\title{
DESIGUALDAD, MOVILIDAD, POBREZA: NECESIDAD DE UNA POLÍTICA SOCIAL DIFERENTE
}

\author{
Claudio Sapelli \\ P. Universidad Católica de Chile
}

\begin{abstract}
Resumen: En Chile, las generaciones más jóvenes tienen una distribución del ingreso sustancialmente más equitativa que las generaciones más viejas. En ellas hay también mayor movilidad social y menor pobreza. Las tendencias gruesas "van bien", pero la política social no se ha adecuado a este nuevo escenario. El artículo discute cómo debiera cambiar el diseño de la política social para tomar en cuenta estos resultados. El autor considera que la política social debe preocuparse centralmente de la pobreza y de la movilidad social. Los nuevos programas deberían, entonces, centrar su diseño en acompañar a las personas en una etapa de sus vidas (durante un episodio de pobreza) y no para siempre (porque no "son" pobres). En consecuencia, deben basarse en el ingreso y no en una ficha de protección social, cualquiera ésta sea. Es necesario reconocer que la pobreza tiene una importante dimensión temporal, hay alta movilidad entre pobres y no pobres y que la política social debe adecuarse a esa realidad, en lugar de representar una traba.
\end{abstract}

Palabras Clave: política social, distribución del ingreso, movilidad social, pobreza, ingreso ético familiar, impuesto negativo al ingreso. RECIBIDO: julio 2013; ACEPTADO: enero 2014.

Claudio SAPelli. Ph. D. en economía, Universidad de Chicago. Profesor del Instituto de Economía de la Pontificia Universidad Católica de Chile. Email: csapelli@, uc.cl. 


\title{
INEQUALITY, MOBILITY, POVERTY: THE NEED FOR A DIFFERENT SOCIAL POLICY
}

\begin{abstract}
In Chile, income is distributed on a substantially more equitable basis among younger generations than older generations. There is a greater social mobility and less poverty in the younger generations. The overall trends "are going well," but social policy has not been adapted to this new scenario. This article discusses how the design of social policy should be changed in order to take into account these findings. The author believes that social policy must focus on poverty and social mobility. The design of new programs should therefore be centered on accompanying individuals in one stage of their lives (during an episode of poverty) and not forever (because these people are not intrinsically poor). Consequently, the program should be based on income and not on the indicators of a welfare survey, whatever its content may be. It is important to acknowledge that there is an important time dimension to poverty and that there is high mobility between the poor and the nonpoor. Social policy should be adapted to that reality instead of representing an obstacle.
\end{abstract}

KEYwords: social policy, income distribution, social mobility, poverty, ethical family income, negative income tax.

RECEIVED: July 2013; ACCEPTED: January 2014.

\section{INTRODUCCIÓN}

n Chile, las generaciones más jóvenes tienen una distribución del ingreso sustancialmente más equitativa que las generaciones más viejas. En ellas hay también mayor movilidad social y menor pobreza que en las generaciones de sus padres. Si bien discutiré aquí evidencia empírica al respecto (que no ha sido publicada en otros lugares), el objetivo central del ensayo es la política pública que se deriva de estas observaciones. O sea, en una primera sección se discuten los datos relevantes y, en una segunda sección, se discute qué política pública debiera seguirse de las realidades que se describen.

En trabajos previos (Sapelli 2011a, 2011b) se utilizan los datos de la encuesta de empleo de la Universidad de Chile para mostrar que, si bien la distribución del ingreso para toda la población puede mostrar cierta estabilidad, los indicadores de desigualdad por cohortes muestran una persistente mejora a partir de aquellos nacidos en la década de los cincuenta. Los resultados con la encuesta Casen serán presentados con algo de detalle en 
este ensayo, pero el hecho de que ambas bases de datos den conclusiones similares le da robustez a esta observación.

El diseño de la política social requiere además de una (breve) reflexión sobre cuáles debieran ser sus objetivos y sobre la forma más adecuada de alcanzarlos. En ese sentido se discuten, como objetivos posibles, la movilidad, la pobreza y la desigualdad. Me detengo un poco más en el tema de la desigualdad en Chile, que parece ser el centro de mucha discusión últimamente y que algunos analistas creen debe ponerse como el objetivo central de la política social. Este ensayo, sin embargo, recomienda que la política social debe preocuparse centralmente de la pobreza y de la movilidad social.

En particular, se recomienda que la política respecto de los pobres tome en cuenta la mayor movilidad que hoy existe, lo que, además, mejorará los incentivos para trabajar. De esta manera, lograremos mejorar el diseño de la política social y al mismo tiempo atenuar sus efectos anticrecimiento.

\section{LA EVOLUCIÓN DE LOS INDICADORES SOCIALES}

La evolución de los indicadores sociales como la distribución del ingreso, la movilidad social y la pobreza en Chile "va bien". Normativamente, sin embargo, uno podría aceptar esto y aun así afirmar que hay mucho por hacer. Decir que nos estamos moviendo en la dirección correcta (y no estancados) es diferente a decir que "llegamos" a algún puerto o que definitivamente "estamos bien".

Esta constatación de que "vamos bien" nace de lo que en la literatura técnica se llama "análisis de cohortes". Éste implica estudiar no la evolución de los indicadores año a año, estimados como promedios de toda la población, sino que estudiar la realidad de las diferentes generaciones por separado. Para ello la población se separa en cohortes o generaciones según el año de nacimiento de las personas. A partir de ahora usaremos el término "generaciones", que es de uso más amplio.

De dicho estudio obtenemos que los indicadores, cuando se estiman por generación, tienen una historia de mejora importante. Conviene recalcar que esta es solamente una forma "diferente" de ver la información, ya que ella es la misma sobre la cual se basan los indicadores promedio que solemos manejar. 


\section{¿Por qué hacer un análisis de generaciones?}

Chile es un país en que ha habido muchos cambios en poco tiempo, pero cuando se toman promedios poblacionales estos cambios a veces se pierden o no se ven tan claros. Pero si uno ordena los datos por generación, sí resulta claro que existe una tendencia a la mejora en los indicadores sociales, tendencia que en varios casos es de larga data.

Un buen ejemplo lo obtenemos de un análisis basado en datos de la OCDE. En el gráfico 1 se observa el porcentaje de personas con educación secundaria por grupo etario, que viene a ser una agrupación generacional, de personas nacidas en años cercanos.

Si uno se fija en el porcentaje promedio (el que agrupa a toda la población, en las últimas tres barras), vemos que Chile tiene un porcentaje de educación secundaria muy inferior a Estados Unidos y algo inferior al de la OCDE. Pero si uno mira los datos por generaciones, vemos que Chile ha estado haciendo importantes progresos, y en la generación de 25 a 34 años ya superó el promedio de la OCDE y está muy próximo al de Estados Unidos.

Un enfoque de este estilo no es tan útil en países donde todas las generaciones tienen similares niveles de educación, como es el caso de Estados

\section{Gráfico 1. PORCENTAJE DE PERSONAS CON EDUCACIÓN SECUNDARIA}

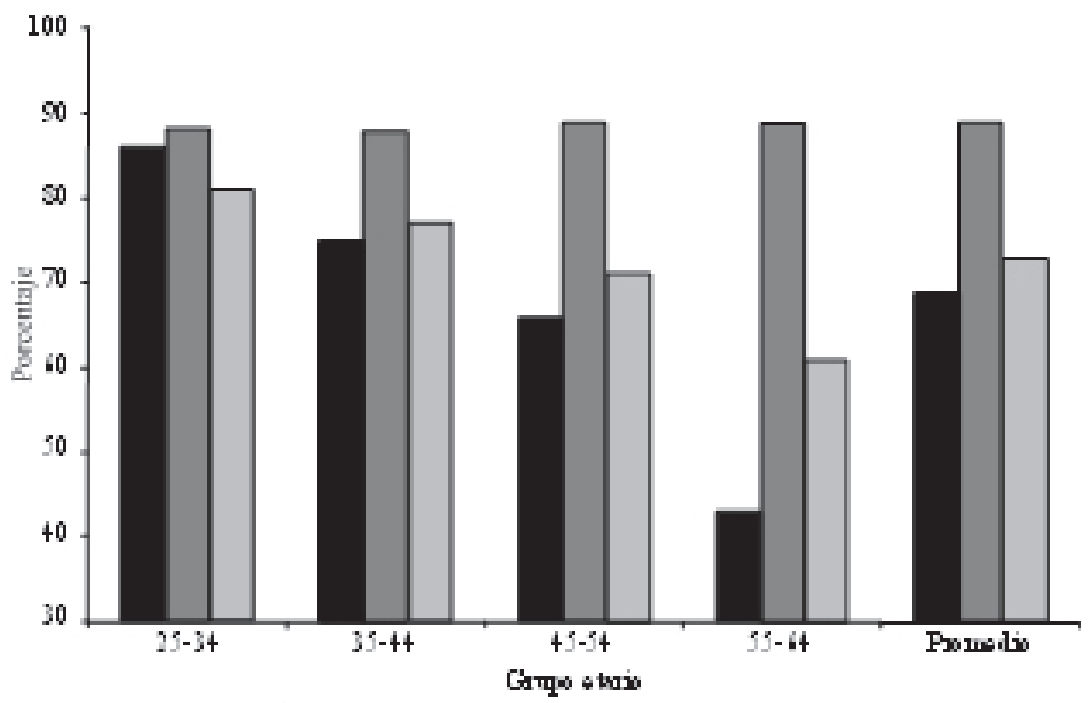

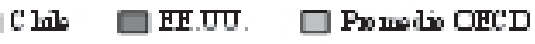

Fuente: Elaboración del autor en base a datos de la OCDE (2011). 
Unidos. Sí lo es en Chile, en donde, si se mira el promedio del país, se podría decir que tenemos un problema de acceso de la población a la educación secundaria, pero si se mira el tema por generaciones se da cuenta que el acceso está ya resuelto en las generaciones más jóvenes. Por lo tanto, la conclusión es que, desde un punto de vista de la política pública, no nos debiera preocupar el acceso a la educación secundaria.

\section{Distribución del ingreso: la visión de siempre y la visión de generaciones}

Este enfoque de generaciones también podemos aplicarlo a la distribución del ingreso. ¿Son las generaciones más viejas muy desiguales pero las más jóvenes no? ¿O son todas las generaciones, desde las más viejas a las más jóvenes, igualmente desiguales?

La frustración que suele palparse en Chile cuando se discute la distribución del ingreso se deriva de mirar los datos del gráfico 2 (con datos de la encuesta de empleo de la Universidad de Chile). Allí tenemos la evolución de la desigualdad del total de la población y su evolución en el tiempo, sin distinguir por generación. En esta perspectiva, el Gini parece oscilar sin

Gráfico 2. GINI POR AÑO DE ENCUESTA: LA VISIÓN DE SIEMPRE

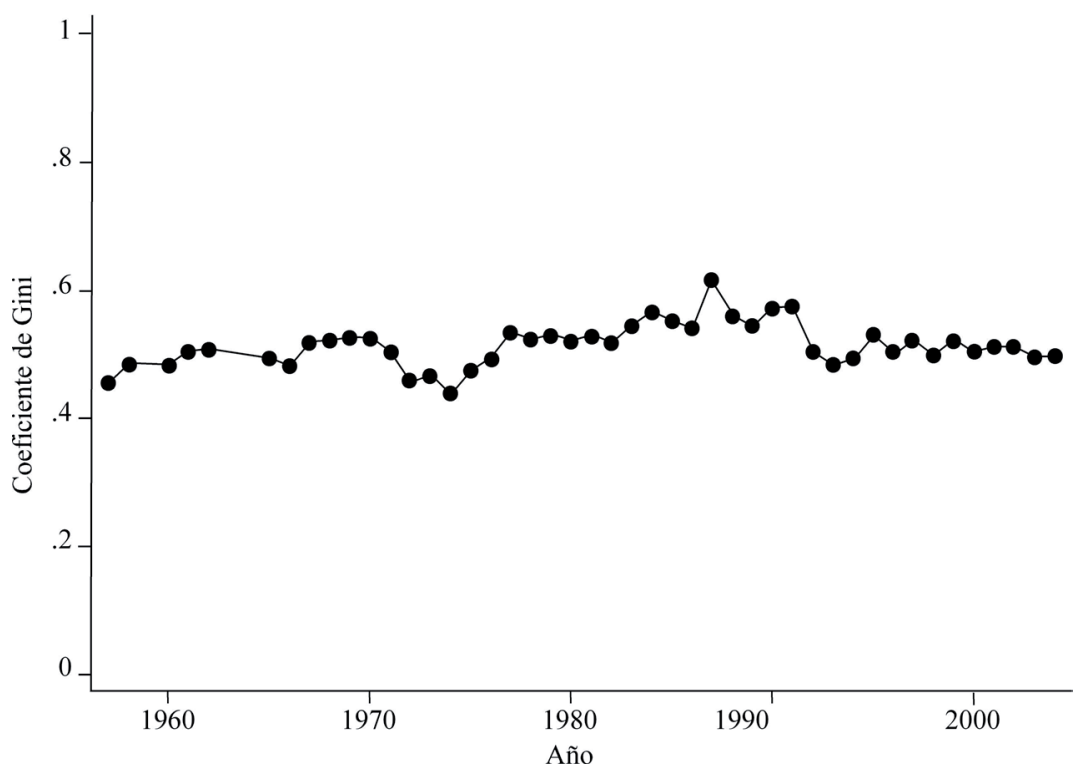

Fuente: Sapelli 2011a, en base a datos de la U. de Chile. 
dirección clara en torno a 0,5 , un valor muy alto. Aunque mirándolo en más detalle, se puede observar que hay una tendencia a la mejora desde fines de los ochenta, que los datos de la Casen ratifican que existe muy marcadamente desde el año 2000 (ver gráfico 8).

Sin embargo, cuando miramos esta evolución por generaciones (gráfico 3), la evolución es muy diferente. La desigualdad empeora hasta los

\section{Gráfico 3. DISTRIBUCIÓN DEL INGRESO POR GENERACIÓN*}

(La linea continua representa el Gini por generación. La línea punteada, la tendencia)

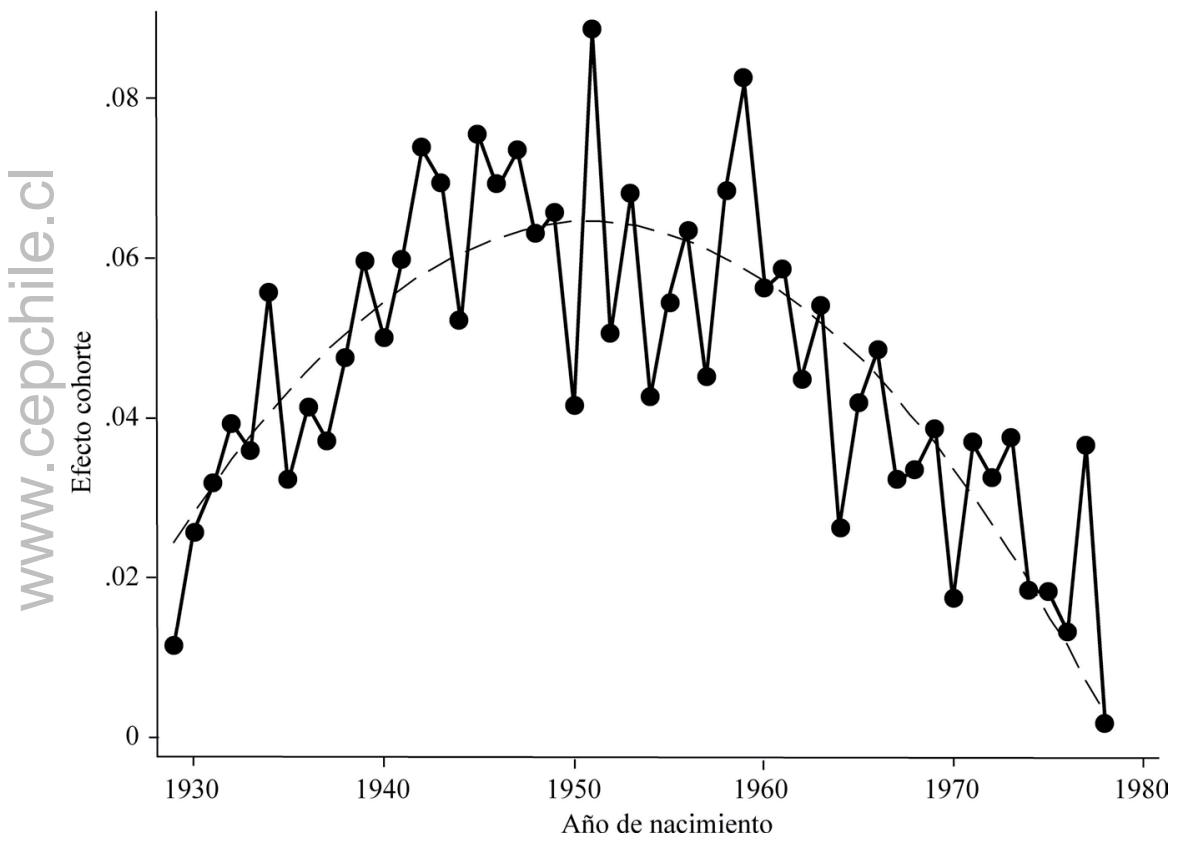

Fuente: Sapelli 2011a, en base a datos de la U. de Chile.

* Lo graficado aquí es el llamado "efecto cohorte" puro que resulta de aplicar el método de Deaton, que permite separar el efecto generación del efecto edad. Como las generaciones nacidas a principio de siglo las observamos solamente cuando son "viejas" y las más recientes, cuando son jóvenes, se requiere de un método para evitar confundir el efecto en su ingreso que es propio de la generación del efecto que es propio de su edad. El método de Deaton es el más utilizado para hacer esta descomposición (ver Deaton \& Paxson 1994; Deaton 1997; Attanasio 1998; Deaton \& Paxson 2000; Blisard 2001; McKenzie 2002). Incluso métodos desarrollados más recientemente (como el del double hurdle) son sólo modificaciones menores del método original (ver, por ejemplo, Aristei et al. 2008). Se excluyen de éste y otros gráficos los intervalos de confianza a efectos de hacerlos más legibles, pero todos los procesos analizados son significativos al $5 \%$ cuando se los analiza punta a punta. 
nacidos a mediados de los cincuenta y a partir de allí empieza a mejorar. La mejora de punta a punta, desde las generaciones nacidas a mediados de los cincuenta hasta las nacidas a principios de los ochenta, es de 8 puntos del Gini, una mejora sustancial. Con los datos de la Casen se obtiene algo similar en términos de evolución (ver discusión más adelante) y el orden de magnitud de la mejora en el indicador de distribución del ingreso es incluso algo mayor: 14 puntos de Gini.

En resumen, si bien el indicador de toda la población indica poca variación, la distribución del ingreso por generaciones ha mejorado sustancialmente. La razón de por qué ello no se ha notado aún en el indicador global tiene que ver con la recomposición de la población chilena, en la que los grupos etarios más viejos, que son los que tienen peor distribución del ingreso, han adquirido mayor peso.

\section{Robustez de la evidencia empírica sobre distribución del ingreso}

Los gráficos 4 y 5 muestran el efecto cohorte calculado sobre el Gini para las encuestas Casen (se utilizan todas las disponibles a la fecha del estudio, desde 1990 hasta 2009). El gráfico 4 muestra con la definición normal de generación (agrupando a todos los nacidos en un año) y el gráfico 5 lo hace calculando el Gini sobre cohortes definidas como un promedio móvil de cinco años (para cada generación se incluyen los datos de los nacidos en ese año más los datos de los nacidos dos años antes y dos años después, una técnica para encontrar resultados más robustos).

Este análisis confirma la caída en la desigualdad por cohorte, la cual se acentúa a partir de los años 50. En términos de magnitud, la estimación con generaciones definidas tradicionalmente es de una caída de 12 puntos y en el caso de las generaciones definidas como promedios móviles, la caída empieza antes y es mayor, de 14 puntos entre la generación nacida en 1943 y la nacida en 1977.

En el gráfico 6 se confirman los resultados, utilizando otros índices para medir la desigualdad, diferentes al Gini. Hay diferencias entre los resultados para el Gini y para los otros índices, pero estos muestran una mejora aún mayor en la desigualdad.

Si se trata de explicar esta mejora de la desigualdad, los datos de las encuestas Casen permiten ratificar que la educación ha jugado un rol importante. En el gráfico 7 se muestra la evolución de un indicador de des- 
Gráfico 4. EFECTOS COHORTE DEL GINI, DEFINICIÓN TRADICIONAL

(La línea continua representa el Gini por generación. La línea punteada, la tendencia)

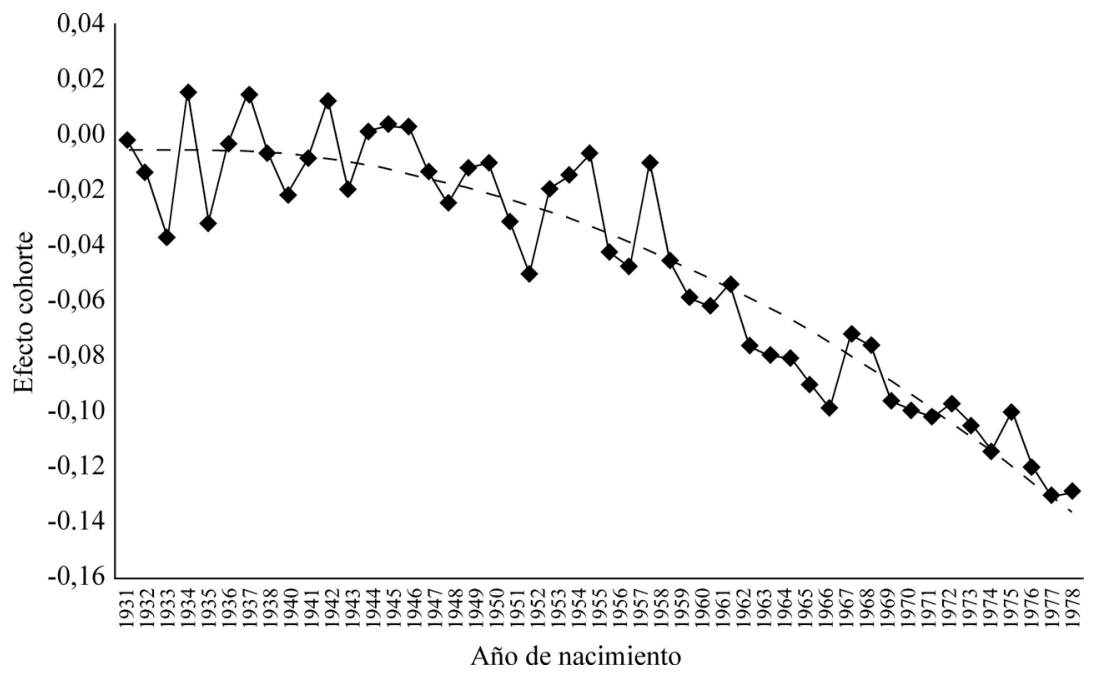

Fuente: Elaboración del autor en base a datos de las encuestas Casen 1990-2009.

Gráfico 5: EFECTOS COHORTE DEL GINI EN PROMEDIOS MÓVILES

(La línea continua representa el Gini por generación. La linea punteada, la tendencia)

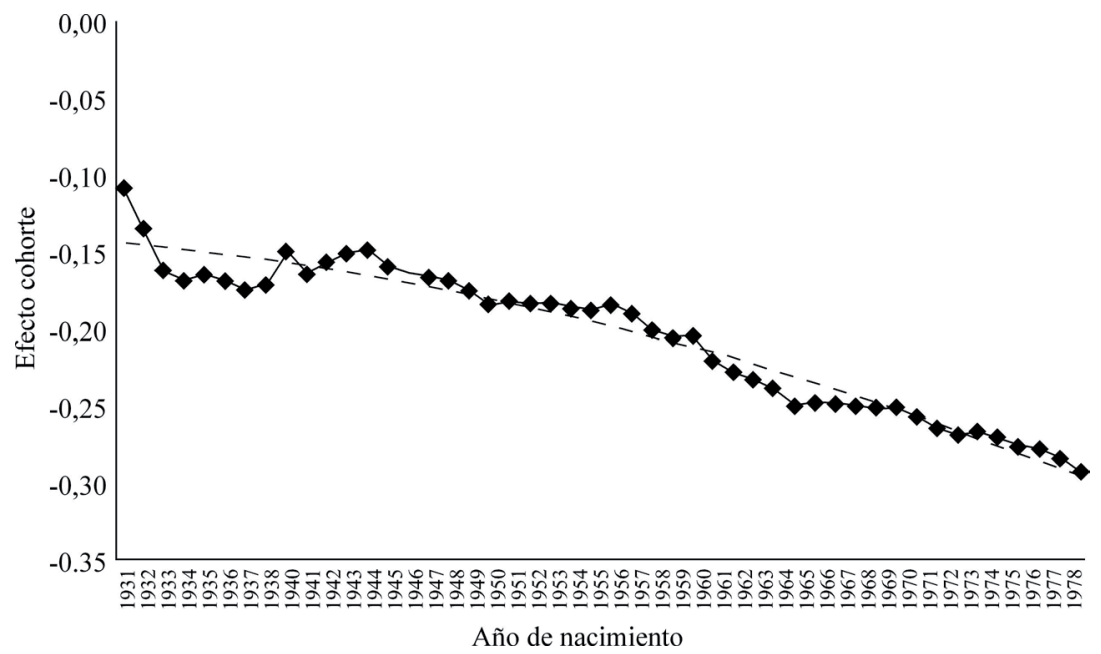

Fuente: Elaboración del autor en base a datos de encuestas Casen 1990-2009. 
Gráfico 6. COMPARACIÓN DEL EFECTO COHORTE ENTRE TRES ÍNDICES DE DESIGUALDAD

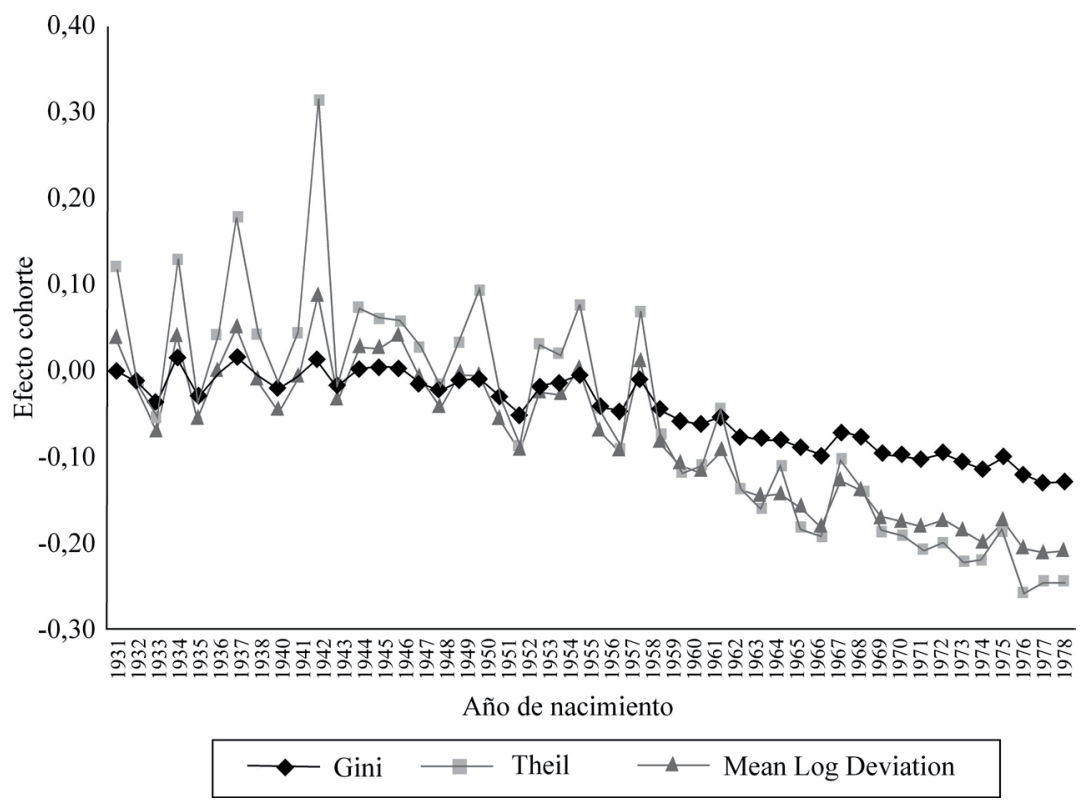

Fuente: Elaboración del autor en base a datos de las encuestas Casen 1990-2009.

igualdad de la educación de las personas en cada cohorte (en que se usa, al igual que para los ingresos, el índice de Gini). Allí puede observarse una persistente disminución de los niveles de desigualdad.

\section{Conclusión}

Las cohortes se han vuelto crecientemente más iguales en términos de distribución del ingreso. La educación ha cumplido un rol fundamental en este proceso. Si bien esta mejora en la distribución del ingreso se observa muy claramente al analizar los datos por generación (tanto a través de las encuestas de la Universidad de Chile como a través las encuestas Casen), esta tendencia ya se nota a nivel de datos totales para la población (gráfico 8). La tendencia es más fuerte para la población en edad de trabajar (18 a 65 años): una mejora de 5 puntos del Gini, casi 10 por ciento en el indicador. 
Gráfico 7. GINI DE LOS AÑOS DE EDUCACIÓN, POR GENERACIÓN

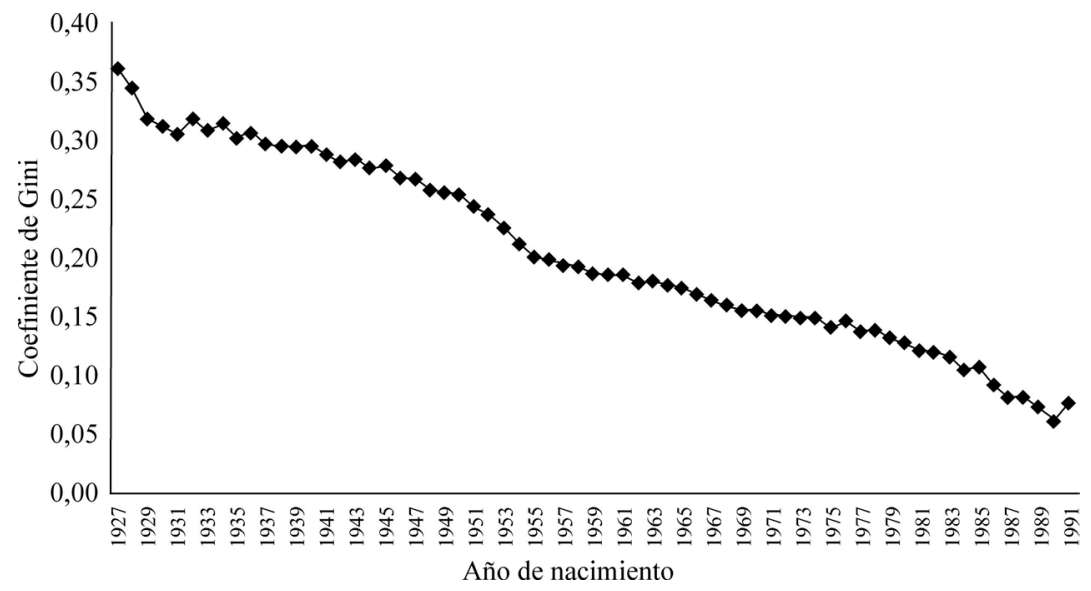

Fuente: Elaboración del autor en base a datos de las encuestas Casen 1990-2009.

Gráfico 8. EVOLUCIÓN DEL GINI PARA LA POBLACIÓN TOTAL

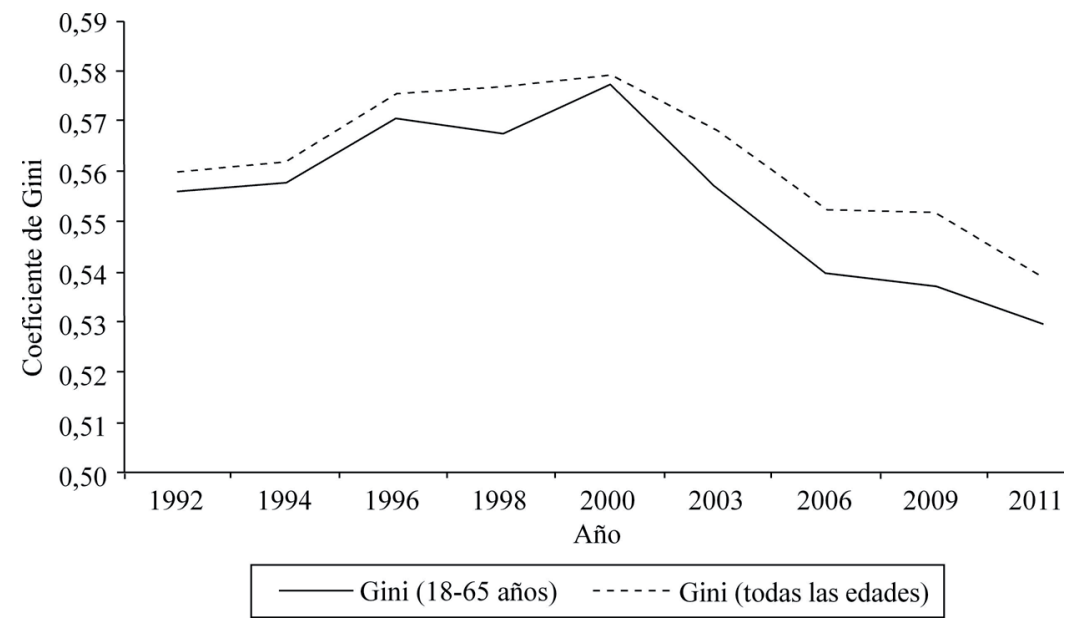

Fuente: Elaboración del autor en base a datos de las encuestas Casen 1992-2011. 
Esta evolución entonces ya no debiera ser un punto de controversia: existe, las mediciones son concluyentes. De manera que respecto de cómo mejorar la distribución del ingreso, la discusión debiera centrarse en si la velocidad de progreso es o no la adecuada y si puede aumentarse sin afectar en exceso el crecimiento. Pero ésta es una discusión diferente a la originada en la frustración, porque el indicador de Gini no muestra mejoras.

En relación con este último punto, es conveniente recordar que la distribución del ingreso es un tema en el que el progreso es lento y en el que, por lo tanto, la impaciencia puede ser mala consejera. Cuando el Banco Mundial armó una base de datos de diferentes países y distintos momentos en el tiempo de indicadores de distribución del ingreso (Deininger \& Squire 1996), uno de los aspectos más notorios de los datos era que, si bien los países tenían diversos niveles de desigualdad, esos niveles permanecían relativamente constantes por largos periodos en todo el mundo.

Tratar de empujar muy fuerte en la desigualdad de ingreso puede terminar minando el proceso de crecimiento, y este último constituye la base de los positivos resultados que Chile ha estado observando. Ellos son, a su vez, la base del aumento de la demanda de personas con más educación y los que, además, han llevado a aumentar significativamente los ingresos de los más educados, hecho que explica, también, que la población se esté educando más. O sea, el crecimiento es el motor de la mayor cantidad de años de educación de la población, de los mejores salarios y del mayor empleo, todo lo cual está detrás de la mejora en la distribución del ingreso en las generaciones más jóvenes.

\section{MOVILIDAD SOCIAL}

Aun cuando hubiéramos concluido algo diferente respecto de la evolución de los indicadores de distribución del ingreso, uno podría argumentar que esta distribución no debiera ser el objetivo último de la política social, sino que este objetivo debiera ser la mayor movilidad social, una distribución de oportunidades más equitativa.

Es relevante entonces responder la pregunta: ¿Qué ha pasado en Chile al respecto? Para ello podemos analizar la evolución de la movilidad educacional intergeneracional (Sapelli 2011c); esto es, cuánto depende la educación del hijo de la educación del padre. Esto se mide por un indicador que va de cero (la educación del hijo es independiente —o sea, no depende 
para nada- de la educación del padre) a uno (la educación del hijo depende totalmente — es idéntica- de la educación del padre). De esto se concluye que en este indicador lo mejor es estar más cerca de cero y, en términos de evolución, lo mejor es que el indicador caiga. Esto es lo que ha sucedido en Chile, como puede observarse en el gráfico 9. Desde las generaciones nacidas en los treinta a aquellas nacidas en los ochenta ha habido una mejora importante en la movilidad (el indicador cayó aproximadamente de 0,7 a 0,4$)$. Si bien éstas son buenas noticias, se ve una cuasi detención del proceso de mejora sobre el final, lo que es preocupante.

Más allá de que el progreso es importante, impacta el estancamiento entre la generación nacida en 1957 y aquella nacida en 1978. Analizando las causas de este estancamiento, se concluye (puede verse la fundamentación en Sapelli 2011c) que un factor importante fue la imposibilidad de acceder a educación universitaria a partir del congelamiento de las vacantes que ocurre a mediados de los 70 y que para la educación universitaria dura 20 años. La generación nacida en 1957 (que es cuando se estanca el proce-

\section{Gráfico 9. MOVILIDAD INTERGENERACIONAL DE LA EDUCACIÓN: EVOLUCIÓN EN} EL TIEMPO

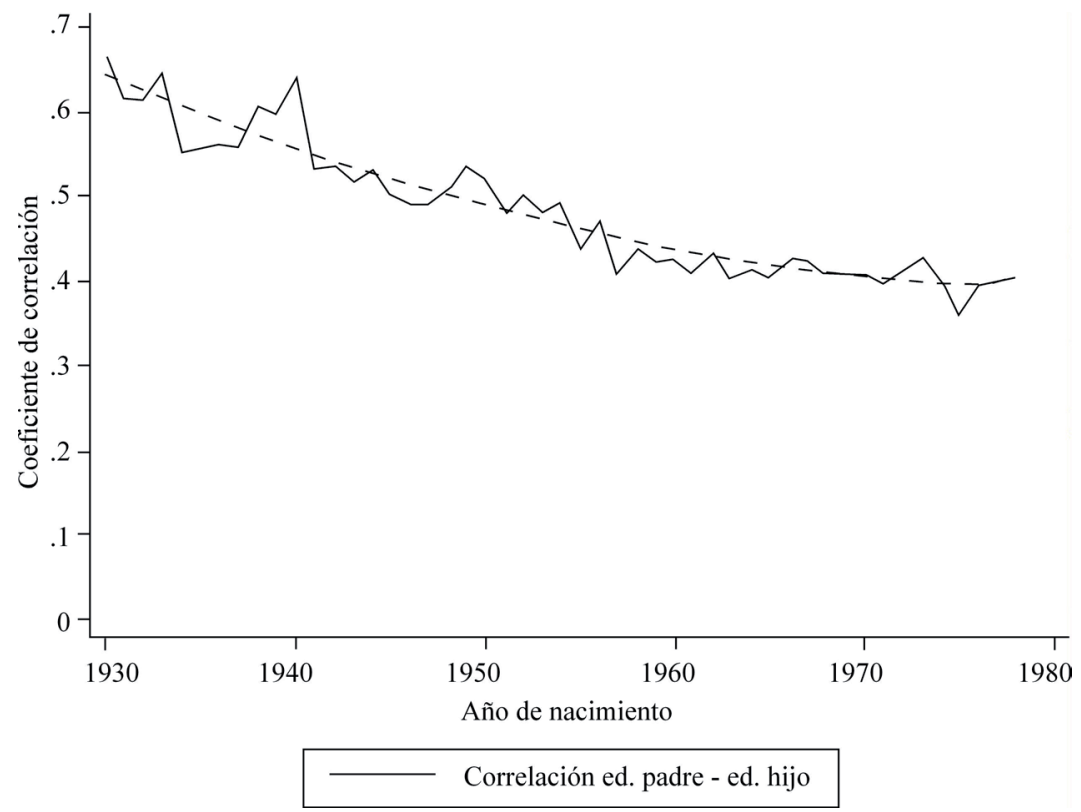

Fuente: Sapelli 2011c. 
so de aumento en la movilidad) debía entrar a la universidad justamente en 1975, pero vio fuertemente restringido su acceso. Esto fue lo que falló. En ese sentido, la información permite observar que la cobertura de educación terciaria para quienes son hijos de padres con educación terciaria mejora en 20 puntos porcentuales entre la generación nacida a fines de los cincuenta y aquella nacida a fines de los setenta. Pero, simultáneamente, puede verse que las curvas de cobertura para hijos de padres con menor educación divergen y en especial la cobertura para aquellos que son hijos de padres con menos de 12 años de educación, que mejora poco y nada en ese periodo. Ahí puede verse que el congelamiento de las vacantes universitarias y el racionamiento de puestos vía Prueba de Aptitud Académica impidieron una mayor movilidad intergeneracional de la educación.

Lo importante es que eso ya ha cambiado. El estancamiento en las vacantes del sistema terciario ha sido superado con el cambio del marco institucional de la educación terciaria, que ocurrió a principios de los 90 y favoreció a las personas nacidas a mediados de los setenta (sobre el final del periodo que pudimos observar). Este cambio es aún demasiado reciente como para poder evaluar su efecto en la movilidad, pero es de esperar que haga que ella mejore en el futuro. Como una reflexión anexa, puede considerarse que la restricción cuantitativa generó importantes premios a la educación terciaria, lo que dio lugar a un aumento excesivo de la oferta, que, al hacer desaparecer muchos de los importantes premios salariales de las distintas carreras, está detrás del descontento actual.

Finalmente, conviene recalcar que este proceso de aumento de la movilidad intergeneracional se ha visto acompañado de un proceso de movilidad intrageneracional importante (Sapelli 2013), que es la razón de que la pobreza hoy deba conceptualizarse como temporal, tema que resulta central en el diseño de una adecuada política social, como se verá más abajo.

\section{POBREZA}

Respecto de la pobreza, utilizando el mismo método de generaciones usado para analizar la distribución del ingreso y la movilidad, se puede observar, en el gráfico 10, una marcada caída de ella. Los datos también muestran que la pobreza es principalmente un tema juvenil. Así, las personas son pobres durante su niñez y, luego, la mayor educación los saca de la pobreza y pueden disociarse de la suerte de sus padres. Pero el ser pobre 
cuando menor de edad deja marcas permanentes en el desarrollo cognitivo, por lo que el apoyo a esa edad es fundamental para que esta mejora no tenga un techo. Por otra parte, la temporalidad de la pobreza se acentúa con el aumento observado en la movilidad social.

\section{5. ¿HAY QUE ELEGIR ENTRE POBREZA Y DESIGUALDAD?}

Por muchos años, el objetivo de la política social chilena ha estado en el combate a la pobreza. Hoy se discute cambiar el énfasis y hacer más esfuerzo en combatir la desigualdad. ¿Un pronunciamiento en favor de una política que promocione la igualdad de ingresos podría significar una atenuación en la fuerza con que se combate la pobreza? ¿Es necesario elegir entre ambos objetivos? ¿Se pueden perseguir ambos al mismo tiempo?

Vamos por partes.

Primero: es importante dejar en claro que una política de combate a la pobreza es, por sí misma, una política de combate a la desigualdad. Todo lo que mejore los ingresos de aquellos más pobres disminuirá la desigualdad

Gráfico 10. POBREZA POR COHORTES

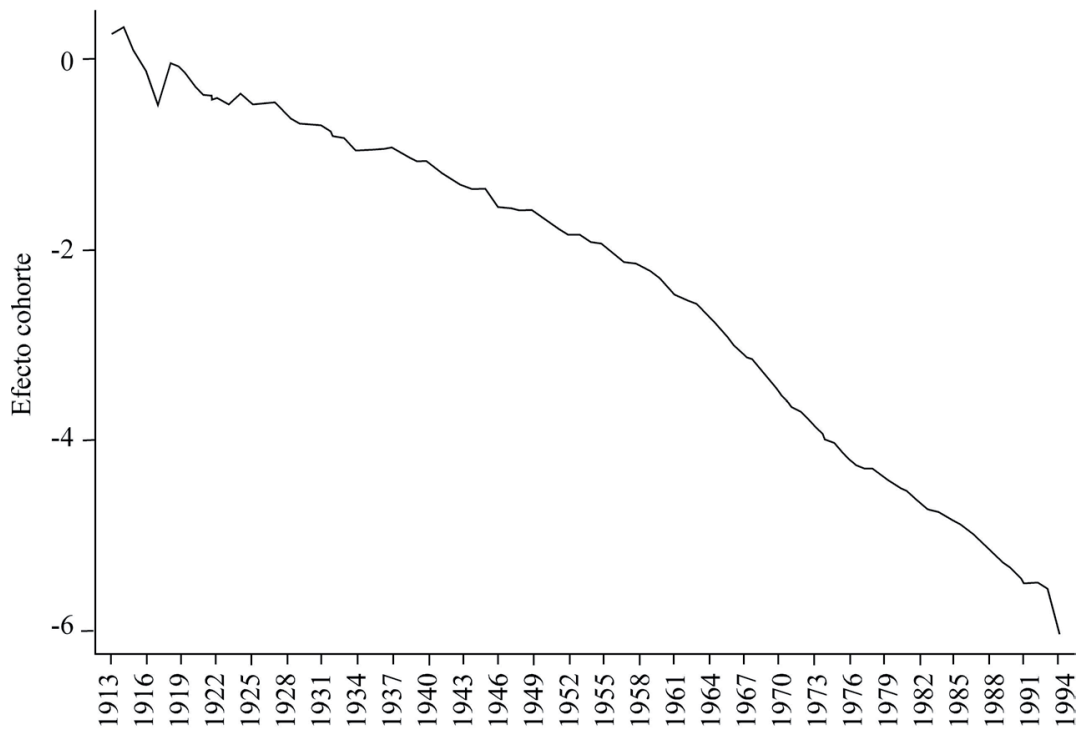

Año de nacimiento

Fuente: Sapelli 2011b. 
de la totalidad del país. O sea, combatir la pobreza es una política complementaria a una política de combate a la desigualdad.

Segundo: frente a los datos recién mostrados, resulta paradójico que en estos momentos surja, y con tanta fuerza, la propuesta de enfocar los esfuerzos en la desigualdad, cuando ella parece estar mejorando sin necesidad de más ayudas que las ya existentes.

Tercero: a mi juicio, combatir la pobreza e incrementar la movilidad son más importantes que reducir la desigualdad. En ese sentido, perseguir un tercer objetivo, por loable que sea, parece inconveniente, especialmente si ello pone en riesgo uno de los dos objetivos principales.

Respondiendo entonces la interrogante del título, me parece que sí, que es necesario elegir entre ambos objetivos de política pública.

Es necesario elegir, en primer lugar, porque cada peso gastado para mejorar la distribución del ingreso en alguien que no es pobre es un peso menos gastado en alguien que sí es pobre.

Veremos que la recomendación de política que se planteará en este ensayo incluye ampliar la base que recibe transferencias, para incluir a los no pobres más cercanos a la línea de pobreza, a quienes podrían aún definirse como "vulnerables". El objetivo principal de ello es eliminar un efecto perverso de la actual política social, que enfrenta a los pobres a impuestos confiscatorios cuando tratan de dejar la pobreza. ¿Qué es lo describimos como impuestos confiscatorios? Estos ocurren cuando una familia deja de ser pobre y sube en un punto del puntaje requerido en la ficha, momento en que se le terminan todos los programas sociales, que obviamente valen más que el ingreso extra que la llevó a superar el límite, con lo cual ese último ingreso, en la práctica, es confiscado.

Una vez que se ha ampliado esta política a aquellos que están cerca de la línea de pobreza se agota la justificación, a mi juicio, para seguir ampliando la cobertura de dicha política. ¿Por qué ayudar a los no pobres que ni siquiera están cerca de la línea de pobreza? ¿Para mejorar la distribución del ingreso? En mi opinión esto no se justifica, básicamente, porque en términos de resultados lo importante es que no haya pobres, que todos tengan un mínimo considerado adecuado y que lo que incluya este mínimo sirva, además, para impedir la transferencia intergeneracional de la pobreza. Para el resto de la población, lo importante es la igualdad de oportunidades y que las políticas no afecten el crecimiento. La política de incluir a los no pobres vulnerables, como veremos, hace que la política social ideada para disminuir la pobreza sea más amigable con el crecimiento. Políticas so- 
ciales universales, en cambio, tendrían importantes efectos sobre la oferta laboral y, por lo tanto, sobre el crecimiento.

La razón de por qué se justifica el ayudar a los no pobres "vulnerables" está en eliminar el fuerte desincentivo a mejorar que existe hoy en la política social chilena.

El problema radica en que la política social chilena actual es un buen mecanismo antipobreza solamente si la pobreza es permanente. Cuando la pobreza es permanente, lo central de la política es identificar a quienes son pobres, razón por la cual se confecciona la ficha de protección social. Como en esa visión las personas estarían condenadas a la pobreza, no habría necesidad de preocuparse de los incentivos que tendría la política social sobre su comportamiento. Entonces, el hecho de que la política enfrente a los pobres a impuestos confiscatorios cuando están por dejar la pobreza no importaría, ya que, bajo este paradigma, ello nunca sucederá.

Sin embargo, como la pobreza se ha transformado en una circunstancia principalmente temporal, el contexto social donde se aplica la política ha cambiado y ésta genera hoy incentivos perversos. La gente sale de la pobreza, algunos para siempre, otros temporalmente. Cualquiera sea el caso, enfrentados a los impuestos confiscatorios estas personas se ven ante el dilema de mentir, de ocultar ingresos o bienes o, incluso, de no mejorar. La política antipobreza, entonces, está diseñada de tal manera que se opone a la movilidad, lo que es un contrasentido.

Por las mismas razones, la ficha de protección social es un mecanismo de focalización que se ha vuelto obsoleto. Ella se basaba en que podía identificarse a un pobre por algunas pocas características permanentes que lo diferenciaban de un no pobre. Pero ahora eso no existe. Ahora un no pobre y un pobre son muy parecidos: sólo difieren en su ingreso. Las personas no "son" pobres, sino que pasan por episodios de pobreza. En ese contexto, el objetivo de la política pública debiera consistir en acompañar a una persona en tanto sea pobre y "soltarla" cuando deje de serlo. Hoy, en lugar de focalizar el gasto social a través de una ficha, es necesario focalizarlo a través del ingreso, que es el único criterio diferenciador entre pobres y no pobres.

$\mathrm{Al}$ mismo tiempo, se debe tener cuidado de que el aumentar en forma importante las transferencias a los no pobres genere incentivos para no trabajar. Es un punto que con un buen diseño del Ingreso Ético Familiar (IEF), no el actual, podría solucionarse.

Por último, en esta dicotomía entre el objetivo actual de la política social (bajar la pobreza) y el objetivo deseado por algunos para el futuro 
(bajar la desigualdad), queda a un lado un tema que, a mi juicio, es clave y que sí debiera ser objeto de un esfuerzo adicional: aumentar la movilidad social o, lo que es lo mismo dicho de otra forma, proveer igualdad de oportunidades.

Si lo que buscamos es la igualdad de oportunidades o la de resultados es una vieja discusión (desde Rawls 1971 a Nozick 1974). Pero lo cierto es que la movilidad es un objetivo importante en el que, además, no hay trade off con la pobreza, ya que hay políticas que permiten perseguir ambos objetivos al mismo tiempo. Por ejemplo, para aumentar la movilidad tenemos que ayudar a los hijos de padres pobres para que no resulten afectados por la pobreza de sus padres. Esto se logra, en parte, mejorando la situación de aquellos padres que se encuentran en situación de pobreza.

\section{RECOMENDACIONES DE POLÍTICAS PÚBLICAS}

La propuesta de este ensayo consiste en agrupar buena parte de las transferencias hoy existentes en una sola política simple y transparente. Esto permitiría convertir al Ingreso Ético Familiar, correctamente implementado, en el pilar central de la política social, lo que ayudaría a mejorar la política antipobreza y, al mismo tiempo, ampliarla a los más cercanos a la línea de pobreza, los aún vulnerables. Este mecanismo contribuiría también a bajar la desigualdad y a aumentar la movilidad.

La proposición es implementar una adaptación del "negative income tax", o impuesto negativo al ingreso (INI), adaptación que también tomaría aspectos prestados del "Earned income tax credit" (EITC) de Estados Unidos. Este programa consiste en "ayudar a quienes se ayudan a sí mismos", subsidiando el ingreso de quienes son pobres pero trabajan, por lo que en la práctica opera como un aumento salarial para estas personas.

Las ventajas de un INI están en que uno puede reemplazar con un programa a gran parte de la multiplicidad de programas sociales que implican transferencias en dinero, lo que simplifica la política social y permite evaluar mejor sus efectos, evitando arbitrariedades y disminuyendo costos de implementación.

Por otra parte, hay ya mucha evidencia empírica acumulada respecto de los efectos del EITC, en particular en Estados Unidos (ver, por ejemplo, a Eissa \& Liebman 1996 o a Meyer \& Rosenbaum 2001). Está visto que mejora los ingresos de los más pobres, disminuye la tasa de pobreza, 
mejora la desigualdad y disminuye la dependencia de las familias de las políticas de bienestar social. También reúne una clara evidencia de un efecto positivo en la participación laboral (en particular sobre las madres solteras), aunque no en las horas trabajadas de quienes ya participaban.

Una adaptación del EITC permitiría, a su vez, implementar a través de un mecanismo alternativo el objetivo del salario mínimo. En otras palabras, a través de una política fiscal — y no de una laboral — se podría asegurar un mínimo de ingresos a la población. Esta política tiene la ventaja de no incurrir en los costos del salario mínimo como lo conocemos hoy, ya que el INI no tiene el efecto perverso de perjudicar la demanda por trabajadores. Aunque puede afectar la oferta, al menos deja en las manos de las personas la decisión de trabajar o no trabajar. El salario mínimo, por oposición, impide que aquéllos con menor capital humano trabajen, aun cuando quieran hacerlo.

A su vez, el INI permite reemplazar a todos los programas que tienen incentivos perversos, como el impuesto confiscatorio ya descrito.

Entre sus ventajas, el impuesto negativo al ingreso permite tratar más simétricamente a pobres y ricos, como se puede ver en el gráfico 11. Allí,

Gráfico 11. IMPACTO DEL IMPUESTO NEGATIVO AL INGRESO

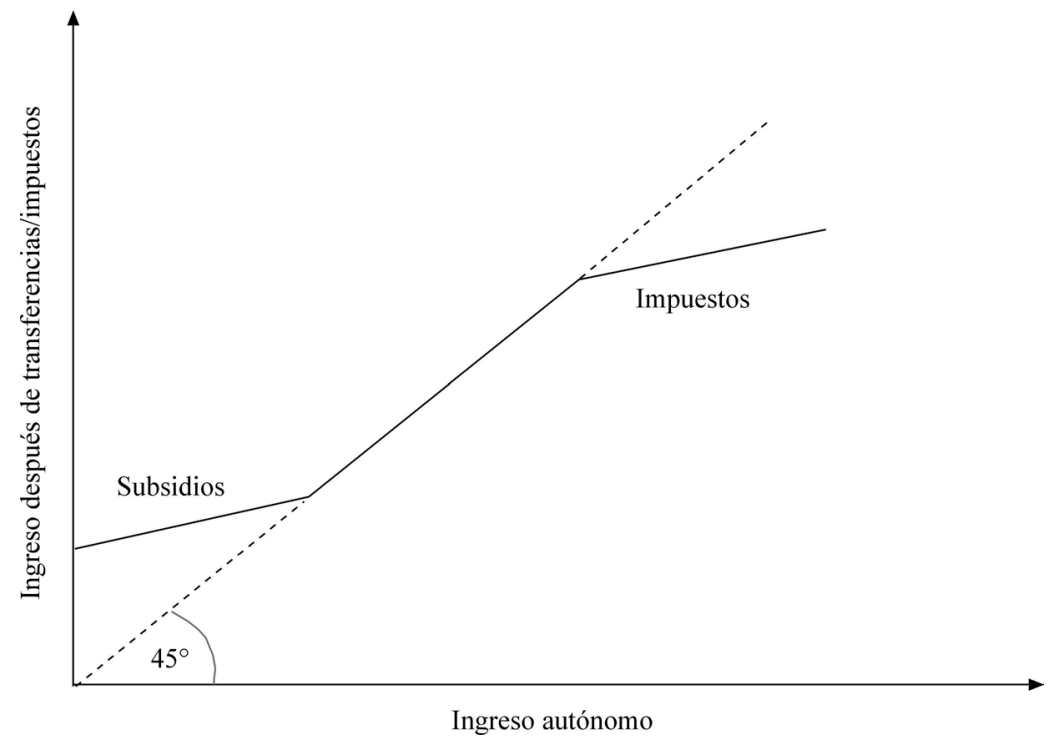

Fuente: Elaboración del autor. 
en el eje horizontal está el ingreso autónomo de la persona y en el vertical, el ingreso después de transferencias e impuestos. La recta de 45 grados significa que no hay diferencias entre ellos. Cualquier política de transferencias, por lo tanto, tiene por objetivo desviarse de la recta diagonal y aumentar los ingresos de los más pobres — vía subsidios — y disminuir los de los más ricos, vía impuestos. Eso es lo que se ve en el gráfico, en que para las personas de menores ingresos, el ingreso después de subsidios es más alto y para las personas de mayores ingresos, el ingreso después de impuestos es más bajo.

El problema es que un esquema de este tipo hay que diseñarlo con mucho cuidado, porque se corre el riesgo de que las personas decidan dejar de trabajar ya que reciben un ingreso suficiente a través de las transferencias. Eso es lo que pasaría, por ejemplo, si se les diera un mínimo no condicionado: todos aquéllos con un ingreso menor al garantizado dejarían de trabajar, dado que si su ingreso es cero recibirán este monto garantizado sin necesidad de laborar. Se daría la tendencia entonces de que toda la cola izquierda de la distribución del ingreso dejaría de contribuir al PIB, viviría de sus rentas y se concentraría en un punto en la distribución, con ingreso autónomo cero, pero ingreso efectivo igual al mínimo garantizado. Como esto requeriría de más impuestos para financiarse y los impuestos generan incentivos perversos, entraríamos en un círculo vicioso.

Una discusión un poco más detallada del impuesto negativo al ingreso, de cómo diseñarlo y su aplicabilidad a Chile, se hace a continuación.

\section{El impuesto negativo al ingreso (INI)}

En esta sección discutiré la recomendación de implementar un impuesto negativo al ingreso (INI). Veré sus ventajas y desventajas, con un ojo puesto en su aplicación a Chile, y terminaré con una discusión de hasta qué punto el Ingreso Ético Familiar se parece al INI.

La propuesta original de Friedman (1962) surge como una alternativa a las políticas vigentes en ese momento en Estados Unidos. A él le preocupaban sobremanera los altos impuestos marginales implícitos en los programas existentes y su enorme desincentivo a trabajar, lo que promovía la creación de una clase dependiente de los ingresos del gobierno y producía un menor nivel de producto.

Los beneficios del INI (B) pueden estimarse por una simple fórmula:

$\mathrm{B}=\mathrm{G}-\mathrm{tY}$, 
Donde $\mathrm{G}$ es el ingreso garantizado, $\mathrm{t}$ es la tasa de impuesto e $\mathrm{Y}$ es el ingreso autónomo de la persona. A una persona con cero ingresos se le garantiza $\mathrm{G}$, y a medida que va creciendo su ingreso autónomo se le va quitando de esta transferencia un porcentaje $t$ de dicho ingreso. A partir de que $\mathrm{G} / \mathrm{t}=\mathrm{Y}$ entonces el beneficio es cero.

Hay dos elementos del diseño que son importantes: el nivel de $\mathrm{G}$ y el de t. Para evitar que el nivel G genere desincentivos a trabajar pareciera necesario que haya condicionalidad, aun cuando Friedman no lo previó en su propuesta original.

Un punto importante de este esquema es que t nunca sea uno (cien por ciento), ya que eso genera los impuestos confiscatorios. El esquema chileno, sin embargo, se parece a un esquema en que t es igual a 1 y que tiene la forma: $\mathrm{B}=\mathrm{G}-\mathrm{Y}$, que justamente posee el incentivo a hacer $\mathrm{Y}=0-\mathrm{o}$ sea, a no trabajar- para todos aquellos que ganan menos que $\mathrm{G}$, o incluso para un pequeño grupo que está por encima de $\mathrm{G}$ y que le gusta el ocio, el incentivo es a no trabajar. En Chile el incentivo puede ser hoy canalizado, más bien, en mentir en la ficha de protección social, pero como ella está siendo cambiada para que sea menos factible hacerlo, el efecto desalentador del sistema puede transformarse en un problema de primer orden.

En cambio, si se deja de cobrar un impuesto expropiatorio de 100\%, se incentiva a las personas a trabajar. Pero los mejores incentivos a trabajar no son las únicas ventajas del INI. También tiene como ventaja que no opera como "un programa especial para pobres", sino que de una manera en años buenos y de otra en años malos. Tiene la particularidad de ser muy flexible y no estigmatizar a las personas. Además, no distorsiona los precios de mercado, como sí hacen otros programas de apoyo, como el salario mínimo o los precios mínimos en la agricultura. El impuesto negativo al ingreso es, a las finales, un mecanismo simple que podría reemplazar a una serie de programas de difícil comprensión y, posiblemente, con menos costos administrativos.

Friedman fue muy crítico del intento de Nixon de implementar un programa que decía estar inspirado en su propuesta. Su principal crítica es que se adicionaba a todos los programas existentes en lugar de reemplazarlos. Esto es algo similar a lo que se puede decir hoy respecto de la implementación del Ingreso Ético Familiar. Al sumar al IEF, como una suerte de INI, al conjunto de programas existentes no se corrige la existencia de impuestos expropiatorios en el margen y, además, se pierden las ventajas de la simpli- 
cidad del INI y sus menores costos administrativos. El INI tiene la ventaja de tratar a los iguales de manera igual, cosa que un conjunto grande y heterogéneo de programas difícilmente puede asegurar.

Permítanme ahora volver al problema de cómo fijar el nivel de $\mathrm{G}$ y $\mathrm{t}$ adecuados. Al poner $\mathrm{t}=1$, como en los programas actuales que existen en Chile, se permite "en principio" focalizar los gastos en los más pobres y proveerlos de una garantía $\mathrm{G}$ mayor (sin por ello generar un gasto público muy grande). Pero, como dijimos, esta configuración tiene el incentivo perverso de que disminuye los incentivos a que estas personas se ayuden a sí mismas trabajando más. Un programa con una menor tasa de impuesto $(\mathrm{t})$ implica gastar más en no pobres y tiene como consecuencia que, si se quiere gastar lo mismo en total, el G garantizado debe bajar. Alternativamente, si se quiere mantener el ingreso garantizado, el gasto total tiene que subir.

El otro punto importante consiste en resolver, en parte, los conflictos que se presentan en diseñar un buen INI al imponer requisitos adicionales para recibir los subsidios. Estos requisitos tratarían de evitar el fraude. Esta es una característica que no estaba en el diseño original del INI pero que sí la tiene el Ingreso Ético Familiar, y es posible que sea necesaria. Por ejemplo, condiciones como exigirles a los beneficiarios que busquen trabajo o que laboren en un esquema público o comunitario. Este tipo de requisitos adicionales pueden resolver el conflicto entre un nivel de ayuda razonable, un impuesto de tasa razonable e incentivos al trabajo razonables.

Ésa es justamente la diferencia entre el impuesto negativo al ingreso y un esquema de tipo "Earned income tax credit" (EITC). El INI da transferencias incondicionadas, en cambio el EITC exige que la persona trabaje. De esta manera, pretende resolver el problema que genera el INI en términos de desincentivar la participación laboral y el empleo.

En el caso del Ingreso Ético Familiar (IEF) hoy aplicado en Chile lo que se está haciendo es continuar con el mal diseño tradicional de la política social chilena, imponiendo impuestos expropiatorios en el margen a los pobres y tratando de resolver el problema de incentivos que ello genera vía otras exigencias. Si bien uno puede jugar en el margen con este trade-off, el IEF es un programa más de transferencias adicionado a los 300 y tantos existentes. Claramente, no es un cambio revolucionario en la política social chilena, como se pensó podía ser.

Sus requisitos, cualquiera sea su forma, están en contradicción con la filosofía del INI propuesto originalmente. En el INI, es el diseño el que pre- 
viene los desincentivos al trabajo, vía una tasa de impuesto no confiscatoria en el margen, y los incentivos remanentes se aceptan como un costo inevitable de la política social.

La idea en la propuesta original del INI es que a quienes no tienen el ingreso suficiente, no se les pregunta por qué no lo tienen. Es decir, el único juicio se hace respecto del nivel de ingreso. Los requisitos exigidos, en cambio, imponen una burocracia para verificarlos y administrarlos, lo que va contra una de las ventajas del INI. Estos requisitos, además, terminan dotando a la política de un grado de discrecionalidad que el INI buscaba remover.

Insisto en que no quiero decir con esto que los requisitos no sean necesarios, sino más bien que disminuyen el atractivo del esquema teórico propuesto originalmente. Hay que ver en la práctica si estos costos no advertidos en el diseño "de pizarrón" son lo suficientemente importantes para que la propuesta de política deje de ser atractiva. En el caso del INI, solamente la implementación del mismo nos permitiría evaluarlo.

Este es un programa, sin duda, en que el diablo está en los detalles. Diseñarlo no es fácil, pero por lo mismo debemos empezar a discutir cómo hacerlo.

Uno de los problemas es que, al igual que en la ficha de protección social, las personas podrían mentir y, en este caso, declarar vivir solas y crear artificialmente un bajo ingreso del hogar (estudiantes universitarios viviendo solos por ejemplo). Otro tema es cómo tratar a personas que pueden ser muy ricas en términos de patrimonio, pero cuyo ingreso es bajo. Para evitar abusos entonces probablemente habrá que recurrir a algún filtro adicional, que requerirá de una burocracia para administrarlo, lo que disminuirá el atractivo que "en el papel" tiene este instrumento.

En todo caso, creo que podríamos concluir que sus beneficios son mayores que los problemas que su implementación genera.

\section{La aplicación del INI en Chile}

¿Qué ha pasado en Chile en los últimos años con la implementación de la política social? ¿Se ha movido en la dirección de un INI? Hay tres áreas en que ello puede evaluarse: mejora de los incentivos para trabajar, operación por reglas en lugar de discrecionalidad y disminución del número de programas. 
Creo que en ninguna de estas tres áreas ha existido en Chile un progreso sustancial. El país continúa teniendo un sistema con altas tasas de impuestos marginales (pese a que últimamente se están haciendo cambios al respecto, subsisten muchos programas anclados en ese esquema). Hay mucha discrecionalidad en lo que al final termina poniéndose en la ficha, ya sea a través de lo que las familias ocultan o a través de lo que convencen al encuestador de ocultar (en cualquier caso, sería sorprendente que el punto de corte de la ficha pudiera efectivamente separar a los pobres de los no pobres. Es una división arbitraria e injusta para aquellos que quedan levemente sobre el puntaje requerido). Finalmente, el número de programas ha crecido y crecido. O sea, Chile se ha mantenido lejos de esta política sencilla, simple, digna.

El INI es además una alternativa para extender beneficios a la clase media baja en una forma saludable, dotando a la política social de adecuados incentivos al trabajo.

El problema quizás pueda estar en los incentivos de la clase política. Dotar a la política de mayor impersonalidad y menor discrecionalidad les quita poder a los políticos. Lo que desde el punto de vista de la sociedad es una de sus ventajas - tener un solo programa, sin discrecionalidad-, puede no serlo desde el punto de vista político.

Agostini et al. (2013) evalúan numéricamente las ventajas de la implementación de un esquema de EITC para Chile y lo comparan con el IEF ya implementado. Concluyen que el EITC es más efectivo en aumentar los ingresos de las personas que están bajo la línea de pobreza y tiene un costo de transferencia por familia menor que el IEF. Concluyen, además, que el EITC promueve la inserción laboral, ya que para las mujeres complementa el salario y contribuye a pagar los costos fijos de salir a trabajar, haciendo más atractivo el mercado laboral para ellas. Finalmente, determinan que el EITC, en el largo plazo, debiera reducir la dependencia del sistema de bienestar social, con lo cual se evita que estas personas caigan en la "trampa de la pobreza" que generalmente producen los programas de transferencias.

\section{CONCLUSIONES FINALES}

Resumiendo, cuando uno mira los datos por generación hay varias tendencias que entusiasman: 
- la distribución del ingreso mejora;

- la movilidad social intergeneracional mejora y, si bien luego se estanca, uno esperaría que continuara mejorando en el futuro, y

- la pobreza mejora.

Sin embargo, también es cierto que la política social no ha sido modificada para estar a la altura de estos cambios. Las tendencias gruesas "van bien", pero la política social no se ha adecuado a todo lo que ha cambiado como consecuencia de ello.

Respecto de las conclusiones de política pública, hay varias que merecen ser destacadas.

En el tema de la pobreza aparece cada vez más relevante su alta dimensión temporal. Es cada día más frecuente que las personas pasen por episodios de pobreza pero que no las convierten en "pobres". Está dimensión temporal de la pobreza es un problema de política pública diferente al problema para el cual se diseñó la actual política antipobreza en Chile. Por lo tanto, es necesario resolverlo con instrumentos diferentes a los usados hasta ahora. En particular, hay que cuidarse de no hacer de la pobreza temporal una permanente, un riesgo que se corre con políticas equivocadas, como sucede cuando ellas crean impuestos expropiatorios en el margen. Preocupa, además, el marco normativo del mercado de trabajo, un problema central de la política social, donde siguen acumulándose cambios que lo hacen menos dinámico (aunque éste es un tema para tratar en otro texto).

El énfasis de la política social debiera estar en promover la movilidad, la igualdad de oportunidades. La política para aliviar las consecuencias de la pobreza puede interpretarse como una política que promueve la movilidad, ya que entre sus beneficios permite que los hijos no sufran las consecuencias de la pobreza de los padres y tengan, entonces, mayores posibilidades de salir de ella. Respecto de la desigualdad, vamos bien encaminados, y no creo que sea necesario hacer más, al menos en el tema de desigualdad por ingresos (el tema de la desigualdad de la calidad de la educación sí merece atención).

Estos cambios que se recomiendan en la política social tienen como consecuencia un aumento en el gasto. La recomendación es agrandar la asistencia en torno a un programa en la lógica de impuesto negativo al ingreso, simple, no discriminatorio, que no tenga impuestos confiscatorios en el margen. Promovería la movilidad, el alivio de la pobreza y disminuiría la desigualdad, mejorando los incentivos a trabajar. 
Para que estén bien diseñados estos programas deben acompañar a las personas en una etapa de sus vidas (durante un episodio de pobreza) y no para siempre (porque no "son" pobres). Implica, por lo tanto, basarlos en el ingreso y no en una ficha de protección social (cualquiera sea ésta). Es necesario reconocer que la pobreza tiene una importante dimensión temporal, que hay alta movilidad entre pobres y no pobres y que, en consecuencia, hay que adecuar la política social a esa realidad. El actual diseño de la política social representa una traba para dicha movilidad. EP

\section{REFERENCIAS}

Agostini, Claudio, Javiera Selman \& Marcela Perticará. 2013. "Una propuesta de crédito tributario al ingreso para Chile". Estudios Públicos 129: 49-104.

Aristei, David, Federico Perali \& Luca Pieroni. 2008. "Cohort, Age and Time Effects in Alcohol Consumption by Italian Households: a Double-hurdle Approach". Empirical Economics 35(1): 29-61.

Attanasio, Orazio. 1998. "A Cohort Analysis of Saving Behavior by U.S. Households". Journal of Human Resources 33: 575-609.

Blisard, Noel. 2001. "Income and Food Expenditures Decomposed by Cohort, Age and Time Effects". Technical Bulletin 1896, United States Department of Agriculture.

Deaton, Angus. 1997. The Analysis of Household Surveys: A Microeconomic Approach to Development Policy. Baltimore: Johns Hopkins University Press.

Deaton, Angus \& Christina Paxson. 1994. "Intertemporal Choice and Inequality". The Journal of Political Economy 102(3): 437-467.

Deaton, Angus \& Chistina Paxson. 2000. "Growth and Saving Among Individuals and Households". The Review of Economics and Statistics 82(2): 212-225.

Deininger, Klaus \& Lyn Squire. 1996. "A New Data Set Measuring Income Inequality". The World Bank Economic Review 10(3): 565-91.

Eissa, Nada \& Jeffrey Liebman. 1996. "Labor Supply Response to the Earned Income Tax Credit". Quarterly Journal of Economics 112(2): 605-637.

Friedman, Milton. 1962. Capitalism and Freedom. Chicago: University of Chicago Press.

McKenzie, David. 2002. "Disentangling Age, Cohort and Time Effects in the Additive Model”. Working Papers 02009. Stanford University, Department of Economics.

Meyer, Bruce \& Dan Rosenbaum. 2001. "Welfare, the Earned Income Tax Credit and the Labor Supply of Single Mothers". The Quarterly Journal of Economics 116(3): 1.063-1.114.

Nozick, Robert. 1974. Anarchy, State and Utopia. New York: Basic Books. 
OECD. 2011. Education at a Glance 2011: OECD Indicators. OECD Publishing. doi: 10.1787/eag-2011-en.

Rawls, John. 1971. A Theory of Justice. Cambridge Mass: Harvard University Press.

Sapelli, Claudio. 2011a. "A Cohort Analysis of the Income Distribution in Chile". Estudios de Economía 38(1): 223-242. 2011b. Chile: ¿Más equitativo? Santiago: Ediciones UC.

2011c. "Sudden Stops in Social Mobility: Intergenerational Mobility in Chile". Documento de Trabajo 400. Instituto de Economía, Pontificia Universidad Católica de Chile.

_ 2013. "Movilidad intrageneracional del ingreso en Chile". Estudios Públicos 131: 1-35. 